\title{
Multidrug-Resistant Candida auris Infections in Critically Ill Coronavirus Disease Patients, India, April-July 2020
}

\author{
Anuradha Chowdhary, Bansidhar Tarai, Ashutosh Singh, Amit Sharma
}

In New Delhi, India, candidemia affected 15 critically ill coronavirus disease patients admitted to an intensive care unit during April-July 2020. Candida auris accounted for two thirds of cases; case-fatality rate was high (60\%). Hospital-acquired C. auris infections in coronavirus disease patients may lead to adverse outcomes and additional strain on healthcare resources.

The ongoing coronavirus disease (COVID-19) 1 pandemic has overwhelmed healthcare systems worldwide. Reports from China and New York have highlighted the concern for nosocomial infections, primarily bacterial, in critically ill COVID-19 patients (1-3). Secondary Candida spp. bloodstream infections in COVID-19 patients with prolonged intensive care unit (ICU) stays have not been documented. However, a new concern coinciding with the brisk expansion of critical care facilities for COVID-19 patients is the potential for nosocomial spread of Candida auris infections (4). C. auris is a global health threat because of its ability to colonize skin, persist in environments, cause nosocomial outbreaks, and lead to severe disease with high mortality rates $(5,6)$.

\section{The Study}

Following up on our prediction (4), we report bloodstream infections caused by multidrug resistant $C$. auris in 1 COVID-19 ICU in New Delhi, India. A total of 596 patients with confirmed COVID were admitted to the 65-bed ICU during April-July 2020. Of these, 420 patients required mechanical ventilation. Overall, candidemia was detected in $15(2.5 \%)$ of the 596 ICU

Author affiliations: Vallabhbhai Patel Chest Institute of the University of Delhi, New Delhi, India (A. Chowdhary, A. Singh); Max Health Care Institute, New Delhi (B. Tarai); International Centre for Genetic Engineering and Biotechnology, New Delhi (A. Sharma)

DOI: https://doi.org/10.3201/eid2611.203504 patients; the predominant agent was C. auris for 10 $(67 \%)$ of those patients. For the remaining 5 patients, candidemia was caused by $C$. albicans $(n=3), C$. tropicalis $(\mathrm{n}=1)$, and C. krusei $(\mathrm{n}=1)$.

We abstracted the following data for the candidemia patients: baseline demographics, medical history, laboratory parameters, microbiological findings, concomitant antimicrobial drug use, and treatments. Isolates were identified by matrix-assisted laser desorption/ionization time-of-flight mass spectrometry (MALDI Biotyper, https:/ / www.bruker.com). In addition, species identification was conducted by amplification and sequencing of the internal transcribed spacer region of ribosomal DNA and of the D1/D2 domain of the large subunit ribosomal DNA. Antifungal susceptibility testing was performed by using the Clinical and Laboratory Standards Institute brothmicrodilution method M27-A3/S4 (7). Antifungals tested were fluconazole, voriconazole, posaconazole, isavuconazole, 5-flucytosine, caspofungin, micafungin, anidulafungin, and amphotericin B.

Most of the 10 patients with $C$. auris infection were elderly ( 8 patients were $66-88$ years of age) and male (7 patients) (Table 1, https:/ / wwwnc.cdc.gov/ EID/article/26/11/20-3504-T1.htm). C. auris was cultured from paired blood samples from all 10 patients and also from urine for 2 of these patients. All of the COVID-19 patients in whom C. auris infections developed had been hospitalized in the ICU for prolonged periods (20-60 days) and had underlying chronic conditions (e.g., hypertension, $\mathrm{n}=7$; diabetes mellitus, $\mathrm{n}$ $=6$; and chronic kidney and liver disease, $n=2$ ). Candidemia caused by $C$. auris developed 10-42 days after admission. Half $(50 \%)$ of the patients with C. auris infections received mechanical ventilation as a result of severe COVID-19 pneumonia. Furthermore, all patients with candidemia had indwelling central lines and urinary catheters. Of the 15 patients, COVID-19 
was hospital acquired for 2 (acquired 2 and 7 weeks after hospital admission). Severity parameters for COVID-19 were elevated for all patients with candidemia (Table 1). Among the 15 candidemia patients, $8(53 \%)$ died; among those with C. auris infection, the fatality rate was $60 \%$. Of note, 4 of the 6 patients who died experienced persistent fungemia, and despite micafungin therapy for 5 days, C. auris again grew in blood culture.

Antifungal susceptibility testing data for C. auris isolates from 10 patients showed that all isolates were resistant to fluconazole (MIC $\geq 32 \mathrm{mg} / \mathrm{L}$ ) and $30 \%$ were nonsusceptible to voriconazole (MIC $\geq 2$ $\mathrm{mg} / \mathrm{L})$. Furthermore, $40 \%$ were resistant to amphotericin B (MIC $\geq 2 \mathrm{mg} / \mathrm{L}$ ) and $60 \%$ were resistant to 5-flucytosine (MIC $\geq 32 \mathrm{mg} / \mathrm{L}$ ). Overall, $30 \%$ of $C$. auris isolates were multiazole (fluconazole + voriconazole) resistant; whereas, $70 \%$ were multidrug resistant, including $30 \%(n=3)$ that were resistant to 3 classes of drugs (azoles + amphotericin B + 5-flucytosine) and 4 that were resistant to 2 classes of drugs (azoles + 5-flucytosine and azoles + amphotericin B). All isolates were susceptible to echinocandins (Table 2, https://wwwnc.cdc.gov/EID/article/26/11/203504-T2.htm).

\section{Conclusions}

Our findings highlight the role of hospital-acquired C. auris bloodstream infections; the patients were probably infected while hospitalized. C. auris can be transmitted in healthcare settings just like other multidrug-resistant organisms, such as carbapenemresistant Enterobacteriaceae and methicillin-resistant Staphylococcus aureus (4). For 4 of 10 patients studied, bacteremia caused by Enterobacter cloacae and Staphylococcus haemolyticus was also noted. In patients with severe COVID-19, the rate of secondary infections was substantially higher, as has been reported by Goyal et al. (6\% of cases of secondary bacterial infections in the United States) (3) and Zhou et al. (15\% of cases of secondary bacterial infections in China) (8). Among fungal co-infections in France, the incidence of putative invasive pulmonary aspergillosis was high $(30 \%)(9)$.

Several major outbreaks of bloodstream infections caused by C. auris have been reported in India, the United Kingdom, Colombia, South Africa, and the United States (5,10-12). In our report, all patients in the ICU had indwelling invasive devices such as central venous and urinary catheters, which may be the source of $C$. auris infections (i.e., candidemia and urinary tract infection). We anticipate that transmission of C. auris to COVID-19 patients by healthcare personnel is unlikely because of the use of personal protective equipment. However, incorrect and extended use of personal protective equipment can lead to self-contamination and transmission.

Of note, 6 of the 10 patients died, possibly because of multiple underlying health conditions. However, $67 \%$ of those who died had persistent candidemia, which may have contributed to their death. Furthermore, multidrug-resistant $C$. auris affects the choice of antifungal therapy and treatment outcomes. Most C. auris isolates are resistant to fluconazole, and panresistant isolates have been described (13). All C. auris isolates in our study were resistant to fluconazole, and $40 \%$ were resistant to amphotericin B, both of which are commonly used in resource-limited countries; therefore, resistance to both classes of drug by C. auris is highly concerning because use of other antifungals such as echinocandins are limited in these countries.

Candidemia affected $2.5 \%$ of the COVID-19 patients in this cohort admitted to the ICU. In a tertiary care center in New Delhi, C. auris was reportedly the second most common Candida species that caused candidemia in non-COVID patients (14). Extensive contamination of the hospital environment has been detected in hospitals experiencing outbreaks of $C$. auris infection, warranting adherence to strict hospital infection prevention practices, such as enhanced cleaning of rooms with chlorine-based disinfectants at high concentrations $(0.5 \%)$ for highly resistant pathogens such as $C$. auris. Critically ill COVID-19 patients with C. auris infection tend to have concurrent conditions (e.g., diabetes mellitus, chronic kidney disease) and risk factors (e.g., need for mechanical ventilation, receipt of steroids). To reduce complications, admission times in overburdened hospitals, and death rates among COVID-19 patients, identifying and treating C. auris infections is vital. A recent report that investigated changes in the fecal fungal microbiomes of COVID-19 patients has shown increasing prevalence of opportunistic fungal pathogens such as C. albicans, C. auris, and Aspergillus flavus (15). These data, along with our findings, provide evidence that the ongoing COVID-19 pandemic may provide ideal conditions for outbreaks of C. auris in hospital ICUs (4). Thus, during the COVID-19 pandemic, extra caution is warranted in hospitals, regions, cities, and countries where $C$. auris is prevalent.

A.C. and A. Sharma drafted the manuscript. A. Singh and B.T. collected the patient details and performed literature searches, identification, and susceptibility testing. All authors read and approved the manuscript. 


\section{About the Author}

Dr. Chowdhary is a clinical microbiologist and a professor at the Vallabhbhai Patel Chest Institute, New Delhi, India. Her main research interest includes fungal infections.

\section{References}

1. Ruan Q, Yang K, Wang W, Jiang L, Song J. Clinical predictors of mortality due to COVID-19 based on an analysis of data of 150 patients from Wuhan, China. Intensive Care Med. 2020;46:846-8. https:/ / doi.org/ 10.1007/s00134-020-05991-x

2. He Y, Li W, Wang Z, Chen H, Tian L, Liu D. Nosocomial infection among patients with COVID-19: a retrospective data analysis of 918 cases from a single center in Wuhan, China. Infect Control Hosp Epidemiol. 2020;41:982-3.

3. Goyal P, Choi JJ, Pinheiro LC, Schenck EJ, Chen R, Jabri A, et al. Clinical characteristics of Covid-19 in New York City. N Engl J Med. 2020;382:2372-4. https://doi.org/10.1056/ NEJMc2010419

4. Chowdhary A, Sharma A. The lurking scourge of multidrug resistant Candida auris in times of COVID-19 pandemic. J Glob Antimicrob Resist. 2020;22:175-6. https:/ / doi.org/ 10.1016/j.jgar.2020.06.003

5. Cortegiani A, Misseri G, Fasciana T, Giammanco A, Giarratano A, Chowdhary A. Epidemiology, clinical characteristics, resistance, and treatment of infections by Candida auris. J Intensive Care. 2018;6:69. https:/ / doi.org/ 10.1186/s40560-018-0342-4

6. Chowdhary A, Sharma C, Meis JF. Candida auris: a rapidly emerging cause of hospital-acquired multidrug-resistant fungal infections globally. PLoS Pathog. 2017;13:e1006290. https://doi.org/10.1371/journal.ppat.1006290

7. Clinical and Laboratory Standards Institute. Reference method for broth dilution antifungal susceptibility testing of yeasts: 4th informational supplement (M27-S4). Wayne (PA): The Institute; 2012.

8. Zhou F, Yu T, Du R, Fan G, Liu Y, Liu Z, et al. Clinical course and risk factors for mortality of adult inpatients with COVID-19 in Wuhan, China: a retrospective cohort study.
Lancet. 2020;395:1054-62. https:/ / doi.org/10.1016/ S0140-6736(20)30566-3

9. Alanio A, Dellière S, Fodil S, Bretagne S, Mégarbane B. Prevalence of putative invasive pulmonary aspergillosis in critically ill patients with COVID-19. Lancet Respir Med. 2020;8:e48-9. https://doi.org/10.1016/S2213-2600(20)30237-X

10. Chowdhary A, Sharma C, Duggal S, Agarwal K, Prakash A, Singh PK, et al. New clonal strain of Candida auris, Delhi, India. Emerg Infect Dis 2013;19:1670-3.

11. Adams E, Quinn M, Tsay S, Poirot E, Chaturvedi S, Southwick K, et al.; Candida auris Investigation Workgroup. Candida auris in healthcare facilities, New York, USA, 2013-2017. Emerg Infect Dis. 2018;24:1816-24. https:// doi.org/10.3201/eid2410.180649

12. Arensman K, Miller JL, Chiang A, Mai N, Levato J, LaChance E, et al. Clinical outcomes of patients treated for Candida auris infections in a multisite health system, Illinois, USA. Emerg Infect Dis. 2020;26:876-80. https://doi.org/10.3201/eid2605.191588

13. Chowdhary A, Prakash A, Sharma C, Kordalewska M, Kumar A, Sarma S, et al. A multicentre study of antifungal susceptibility patterns among 350 Candida auris isolates (2009-17) in India: role of the ERG11 and FKS1 genes in azole and echinocandin resistance. J Antimicrob Chemother. 2018;73:891-9. https://doi.org/10.1093/jac/dkx480

14. Mathur P, Hasan F, Singh PK, Malhotra R, Walia K, Chowdhary A. Five-year profile of candidaemia at an Indian trauma centre: high rates of Candida auris blood stream infections. Mycoses. 2018;61:674-80. https:/ / doi.org/ 10.1111/myc. 12790

15. Zuo T, Zhan H, Zhang F, Liu Q, Tso EYK, Lui GCY, et al. Alterations in fecal fungal microbiome of patients with COVID-19 during time of hospitalization until discharge. Gastroenterology. 2020 Jun 26 [Epub ahead of print]. https:/ / doi.org/10.1053/j.gastro.2020.06.048

Address for correspondence: Pr. Anuradha Chowdhary, Department of Medical Mycology, VP Chest Institute, University of Delhi, New Delhi 110007, India; email: dranuradha@hotmail.com 